\title{
A Practical Approach to Joint Network-Source Coding
}

\author{
Nima Sarshar and Xiaolin Wu \\ Department of Electrical and Computer Engineering \\ McMaster University, Hamilton, Ontario, Canada \\ sarshan@mcmaster.ca/xwu@ece.mcmaster.ca
}

\begin{abstract}
We are interested in how to best communicate a real valued source to a number of destinations (sinks) over a network with capacity constraints in a collective fidelity metric over all the sinks, a problem which we call joint network-source coding. It is demonstrated that multiple description codes in conjunction with proper diversity routing provide a powerful solution to joint network-source coding. A systematic optimization approach is proposed. It consists of optimizing the network routing given a multiple description code and designing optimal multiple description code for the corresponding optimized routes.
\end{abstract}

\section{Introduction}

\subsection{Joint Network-Source Coding: Problem Formulation}

Joint network-source coding (JNSC) is the problem of communicating and reconstructing a (usually real valued) source in a network to a maximal collective fidelity over a given set of sinks, while the flows of the code streams satisfy the edge capacities of the network. JNSC can be considered as a lossy version of the (lossless) network coding problem, since the reconstruction is not necessarily perfect. The source is "observe" by a subset of nodes in the network, called source nodes. Due to capacity constraints, source nodes have to communicate a coded version of the source to their neighboring nodes. Just as in lossless networked coding, intermediate nodes can in general transcode data received from other nodes, and communicate it to their neighbors. Any node in the network, based on the information it receives about the source, can reconstruct the source with some distortion.

Unlike its lossless counterpart, the interaction of lossy source-network codes with arbitrary networks is largely unexplored. In fact, the term network coding refers, almost exclusively, to lossless network communication. This is despite the fact that arguably the majority of applications, both in the Internet and in various wireless setups, involve lossy source communication, in particular for multimedia applications. It should however be noted that some of the well studied examples of multi-terminal source coding problems (e.g., multiple-description coding) are simple examples of a general lossy networked coding problem.

In this paper the network model is similar to, now standard, models in network coding [1]. The JNSC problem is defined by the following elements:

(1) A directed graph $G\langle V, E\rangle$.

(2) A source $X$ in some alphabet $\Gamma$ and a set of distortion measures $\rho^{n}: \Gamma^{n} \rightarrow \mathbb{R}^{+}$. We assume $X$ admits a rate-distortion function $D_{X}($.$) , with \rho$ as the measure.

(3) A function $R: E \rightarrow \mathbb{R}^{+}$that assigns a capacity $R(e)$ to each link $e \in E$. We normalize bandwidth with the source bandwidth, therefore, $R(e)$ is expressed in units of bits per source symbol.

(4) Two sets $S, T \subseteq V$ that denote the set of source and sink nodes respectively. The source 
nodes observe, encode, and communicate $X$ in the network. Source nodes are assumed to be able to collaborate in encoding. This can model, for example, computer networks where sources are encoded off-line and copies of the code are distributed to the source nodes.

Nodes can communicate with neighbor nodes at a rate bounded by the capacity of the corresponding link. The goal is to communicate the source $X$ from the source nodes in $S$, and reconstruct $X$ at the sink nodes in $T$. A distortion vector $\mathbf{d}=\left(d_{t}, t \in T\right) \in \mathbb{R}^{|T|}$ is said to be achievable if $X$ can be reconstructed with a maximum distortion of $d_{t}$ at a sink node $t$ by using a coding scheme that respects the capacity constraints on the links, i.e., the rate of information per source sample communicated over $e$ is less than $R(e)$. As in [1], we need to leave the details of the code unspecified, because it proves extremely hard to come up with the most general class of possible codes. An intriguing problem is how to characterize the set of all achievable distortion $t$-tuples $\mathcal{D}_{X}(G, S, T, R) \subset \mathbb{R}^{|T|}$. In this paper, on the other hand, we are interested in a coding schemes that minimizes a weighted average distortion over all the sink nodes, that is, $\sum_{t \in T} p_{t} d_{t}$ for some weighting vector $\mathbf{p}=\left(p_{t} ; t \in T\right)$.

\subsection{Multiple-Description Coding: a Tool for JNSC}

Multiple-description codes (MDC) have always been associated with robust networked communications, because they are designed to exploit the path and server diversities of a network. The present active research on MDC is driven by growing demands for real-time multimedia communications over packet-switched lossy networks, like the Internet. With MDC, a source signal is encoded into a number of code streams called descriptions, and transmitted from one or more source nodes to one or more destinations in a network. An approximation to the source can be reconstructed from any subset of these descriptions. If some of the descriptions are lost, the source can still be approximated by those received. This is why there seems to be a form of consensus in the literature in that multiple description codes should only be used in applications involving packet loss, because only in this case the overhead in the communication volume can be justified.

This paper shows, however, that MDC is beneficial for lossy communication even in networks where all communication links are error free with no packet loss. In this case, multiple description coding, aided by optimized routing, can improve the overall rate-distortion performance by exploiting various paths to different nodes in the network. This is best illustrated through some examples.

\subsubsection{Example 1}

In Fig. 1, a source node (node 1) feeds a coded source into a network of four sink nodes (nodes 2-5). The goal is to have the best reconstruction of the source at each of these four nodes. All link capacities are $C$ bits per source symbol. MDC encodes the source into two descriptions (shown by solid and dashed boxes in the figure), each of rate $C$. Descriptions 1 and 2 are sent to nodes 2 and 3 respectively. Node 2 in turn sends a copy of description 1 to nodes 4 and 5 , while node 3 also sends a copy of description 2 to nodes 4 and 5 . In the end, nodes 4 and 5 will each receive both descriptions, while nodes 2 and 3 will only receive one description.

To see how the nodes in the network benefit from MDC, let $D_{1}(C), D_{2}(C), D_{12}(C)$ be the distortion in reconstructing the source given description 1 or 2 or both. Let $\mathbf{d}=\left(d_{2}, d_{3}, d_{4}, d_{5}\right)$ be the vector of the average distortions in reconstructing the source at nodes 2 through 5 . Therefore, $\mathbf{d}=\left(D_{1}(C), D_{2}(C), D_{12}(C), D_{12}(C)\right)$.

Let's define $\mathcal{D}_{M}$ as the set of all achievable distortion 4-tuples d. Although MDC is in general a special form of lossy networked coding, $\mathcal{D}_{M}$ still contains a large and interesting subset of all achievable distortion tuples. In this example, it includes for instance, the distortion region achievable by separate source and networked coding. By results in [1], the maximum 


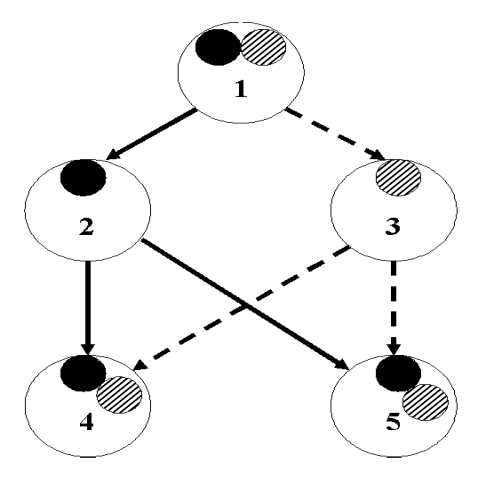

Figure 1: An example of flow of a two description code.

rate with which common information can be communicated to nodes 2 through 5 is $C$ bits per source symbol. Therefore, the distortion rate achievable by separate source and network coding is $\mathcal{D}_{S}=\left\{\left(\delta_{2}, \delta_{2}, \delta_{4}, \delta_{5}\right): \delta_{i} \geq 2^{-2 C}, i=2,3,4,5\right\}$. We immediately have that $\mathcal{D}_{S} \subset \mathcal{D}_{M}$ by noting that $D_{1}(C)=D_{2}(C)=D_{12}(C)=2^{-2 C}$ is part of $\mathcal{D}_{M}$. In fact this corresponds to communicating two identical descriptions, each of which is an optimal (in the rate-distortion sense) source code of rate $C$ for $X$.

The inefficiency of separate source and network coding lies in that even though nodes 4,5 have twice the incoming capacity compared to nodes 2,3 , their reconstruction error $\left(d_{4}=d_{5}\right)$ is bounded by the reconstruction error of the weaker nodes $\left(d_{2}=d_{3}\right)$. Unlike lossless coding, lossy codes can play a tradeoff between the reconstruction errors at different nodes, generating a much larger set of achievable distortion tuples $\mathbf{d}$ than $\mathcal{D}_{S}$. These tradeoffs are essential in practice. For instance, in networked multimedia applications over the Internet, where the network consists of a set of heterogenous nodes, the experience of a user with broadband connection should not be bounded by that of a user with a lesser bandwidth. Such tradeoffs are perhaps best treated as an optimization problem by introducing appropriate Lagrangian multipliers (or weighting functions). An objective function to minimize, therefore, can be defined as

$$
\bar{d}(\mathbf{p}, \mathbf{d})=\mathbf{p}^{T} \cdot \mathbf{d}
$$

where $\mathbf{p}=\left(p_{2}, p_{3}, p_{4}, p_{5}\right)$ is an appropriate weighting vector. An optimal solution will be given by:

$$
\bar{d}^{*}(\mathbf{p})=\min _{\mathbf{d} \in \mathcal{D}_{M}} \bar{d}(\mathbf{p}, \mathbf{d})
$$

Once the optimal distortion vector $\mathbf{d}^{*}$ is found, one should, in principle, be able to find a multiple description code that provides the marginal and joint distortions corresponding to $\bar{d}^{*}(\mathbf{p})$ (such an MDC exists).

As a concrete example, let's optimize the average distortion at all nodes 2 through 5 in Fig. 1 for $\mathbf{p}=(1 / 4,1 / 4,1 / 4,1 / 4)$, in which case:

$$
\bar{d}=\frac{2 D_{12}(C)+D_{1}(C)+D_{2}(C)}{4}
$$

To be specific, lets assume that the source in question is an iid Gaussian with variance one for which achiveable distortions in multiple description coding are completely derived by Ozarow in [2]. The symmetry in indices 1 and 2 ensures that (2) is minimized when the two descriptions 
are balanced, that is, $D_{1}(C)=D_{2}(C)=D$. Ozarow's result, when specialized to balanced MDC states that the following set of distortions are achievable:

$$
\begin{aligned}
D_{1} & =D_{2}=D \geq 2^{-2 C} \\
D_{12} & \geq \frac{2^{-4 C}}{\left(D+\sqrt{D^{2}-2^{-4 C}}\right)\left(2-D-\sqrt{D^{2}-2^{-4 C}}\right)}
\end{aligned}
$$

The average distortion in (2) can therefore be minimized under the constraints of (3). This is a particularly easy task because the region (3) is convex. Let this optimal average distortion be $\bar{d}_{M}^{*}(C)$. By separating source from network coding, the reconstruction distortion at nodes 2 through 5 (and hence the average distortion over all these nodes) is at best $d_{S}(C)=2^{-2 C}$. It is easy to show that $\bar{d}_{M}^{*}(C)<d_{S}(C)$ for all $C>0$. In other words, for all $C>0$ there exists a balanced two description code for which the average distortion over all sink nodes is strictly less than the average distortion achievable by any separate source and network coding scheme.

\subsubsection{Example 2}

As a perhaps more involved example, consider the problem depicted in Fig. 2. A source at node $S$ is to be communicated to nodes $1-8$. The goal again is to minimize the average distortion over all these 8 sink nodes. All links have capacity $C$ bits per source symbol. We choose to use an MDC with 3 descriptions each of rate $C$. These descriptions are indicated by three colors, Solid, Dashed and Diamond. Fig. 2 shows a routing strategy that delivers these descriptions optimally to all the 8 nodes. The routing is optimal since each node receives a number of distinct descriptions exactly equal to its incoming capacity.

Once the routing is optimized, one still needs to optimize the MDC. Unlike the case of two descriptions, there is no closed form representation of the rate-distortion behavior of a balanced 3-description code even in the case of a Gaussian source. Therefore, we resort to the practical technique of Priority Encoding Transmission (PET) for producing whatever required number of balanced descriptions. The class of PET codes are easily parameterizable.

In particular, as is shown in Section 3, for any progressively refinable source with distortionrate function $D(R)$, an MDC of $K$ descriptions each of rate $r$ per packet can be constructed using PET such that the distortion given any $k \leq K$ of the descriptions, the source can be reconstructed with distortion at most $D\left(r \sum_{l=1}^{k} l\right)$, where $\mathbf{y}=\left(y_{l} ; l=1,2, \ldots, K\right)$ is any positive vector such that $\sum_{l=1}^{K} y_{l}=1$. There is a one-to-one correspondence between an MDC code and any such vector $\mathbf{y}$. Optimizing the MDC within the class of PET codes will result into a very convenient convex optimization problem. For the example in Fig. 2, three, four and one nodes receive 1,2,3 distinct descriptions respectively. For a PET code with vector $\mathbf{y}=\left(y_{1}, y_{2}, y_{3}\right)$ the average distortion at the sinks, assuming a Gaussian source of variance one, can be written as:

$$
\bar{d}=8^{-1}\left(3 \cdot 2^{C y_{1}}+4 \cdot 2^{C\left(y_{1}+2 y_{2}+3 y_{3}\right)}+2^{C\left(y_{1}+2 y_{2}+3 y_{3}\right)}\right)
$$

Minimizing the above over all $y_{1}, y_{2}, y_{3} \geq 0$ such that $y_{1}+y_{2}+y_{3}=1$ is a convex optimization problem with linear constraints. For $C=1$, the optimal solution is $y_{1}=0.82, y_{2}=0.18, y_{3}=0$.

\subsection{Design Issues}

Above examples make a number of important observations and expose some design issues, which are the subject of the rest of this paper.

- MDC routing can exploit path diversity in ways that a separate source and network coding can not. For instance, in the example of Fig. 1, nodes 4 and 5 can benefit from the data received both from nodes 2 and 3, while nodes 2 and 3 themselves can benefit 

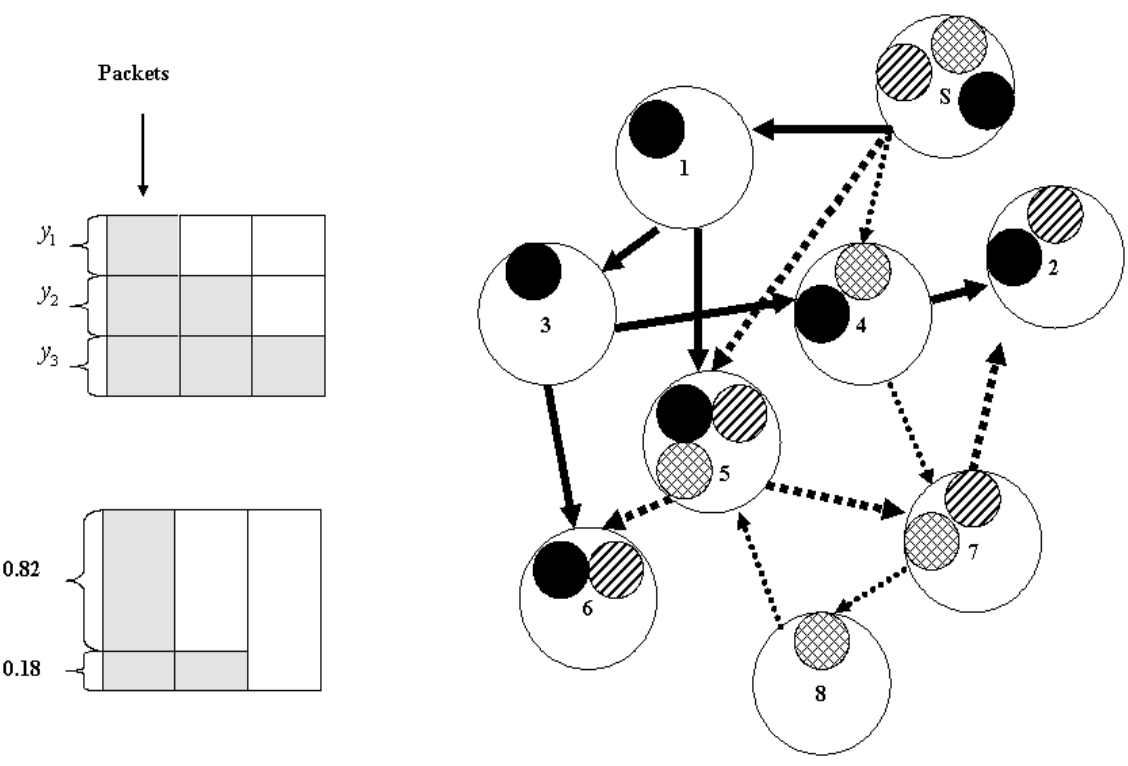

Figure 2: An example of optimal flow of a three description code. The left column of the figure depicts the procedure of designing a 3-description MDC using Priority Encoding Transmission technique as explained in Section 3.

from the data they relay, which was not possible if a common data was communicated to both nodes 2 and 3 by the source node.

- To benefit from MDC in the network, routing needs to be optimized.

- Not only the routing, but also the MDC should be designed optimally. Our strategy will be to optimize MDC generated through PET.

- Although in the above two examples, optimizing the routing of MDC descriptions and optimizing the MDC code happen to be separable, the two optimizations in general, should be carried out jointly. Due to complexity concerns, our strategy is to carry out the optimizations separately.

- Unlike the two examples reported in this section, optimizing the MDC may result in a different number of, potentially unbalanced, descriptions. In this paper, we confine ourselves solely to the case of balanced MDC of the same rate. The total number of descriptions and their rates however, are left as optimization parameters.

In the next section, we consider the problem of optimal diversity routing of MDC packets, called Rainbow Network Flow (RNF) problem. MDC codes designed through PET is introduced in Section 3 where we discuss JNSC through optimized diversity flow and optimal MDC design. Section 4 examines the issues regarding the choice of description rates and the total number of descriptions, and presents simulation results for a family of randomly generated directed acyclic network graphs.

\section{Rainbow Network Flow Problem}

Rainbow Network Flow (RNF) introduced in $[5,6]$ is the problem of optimal routing of MDC packets in a general network. RNF is different from usual commodity flow in that one should 
take into account the information content (or color) of the descriptions. In particular, receiving duplicate descriptions is not beneficial in reconstructing the source. Also, unlike commodity flow, information packets can be duplicated at intermediate nodes. A node desires the rainbow effect by having as many distinct descriptions (or colors) as possible.

A version of the RNF that is particularly relevant to our problem is defined with following inputs:

(1) $G\langle V, E\rangle$, a directed graph with a node set $V$ and an edge set $E$.

(2) $S=\left\{s_{1}, s_{2}, \ldots, s_{|S|}\right\}, T=\left\{t_{1}, t_{2}, t_{3}, \ldots, t_{|T|}\right\}$ two subsets of $V$ representing the set of source and sink nodes respectively.

(3) A function $R: E \rightarrow \mathbb{R}^{+}$representing the capacity of each link in $G$.

(4) A set $\chi \subset \mathbb{N}$ called the description set.

(5) An $r \in \mathbb{R}^{+}$called the description rate.

(6) $\delta:\{1,2, \ldots,|\chi|\} \rightarrow \mathbb{R}^{+}$, a non-increasing function specifying the choice of balanced MDC. $\delta(k)$ is the reconstruction distortion when any subset of size $k$ out of $K=|\chi|$ possible descriptions are present at the decoder.

(7) $\mathbf{p}=\left(p_{t} ; t \in T\right)$ a positive vector that weighs the importance of each sink node $t \in T$.

RNF problem in its most general form allows for each source node $s$ to have any arbitrary subset of the description set $\chi$ (rather than the entire set $\chi$ as the above formulation). A special and important form of the RNF problem is when $\delta(\mathcal{M})=1-|\mathcal{M}| /|\chi|$ for any $\mathcal{M} \subset \chi$. In this case, the distortion is simply given by the size of the subset of available descriptions. Since $\delta(\mathcal{M})$ depends only on the cardinality of the set $\mathcal{M}$, this particular RNF problem is called cardinality RNF (CRNF).

The goal of RNF problem is to find routing (or flow) paths that take descriptions from source nodes to sinks in a way that minimizes a weighted average distortion at the sink nodes.

A flow path from $s \in S$ to $t \in T$ is a sequence of edges $w(s, t)=\left[\left(v_{0}=s, v_{1}\right),\left(v_{1}, v_{2}\right), \ldots\right.$, $\left.\left(v_{m-1}, v_{m}=t\right)\right]$, such that $\left(v_{i}, v_{i+1}\right) \in E$ for $i=0,1, \ldots, m-1$.

A rainbow network flow, denoted by $\alpha(W, f)$, consists of a set $W$ of flow paths in $G$, and a so-called flow coloring function $f: W \rightarrow \chi$ that assigns a description (or color) in $\chi$ to each flow path. For the flows in Fig. $1, W=\{[(1,2),(2,4)],[(1,2),(2,5)],[(1,3),(3,4)]$, $[(1,3),(3,5)]\}$, and the flow coloring function $f$ assigns $f([(1,2),(2,4)])=1, f([(1,2),(2,5)])=$ $1, f([(1,3),(3,4)])=2, f([(1,3),(3,5)])=2$.

Let $\Phi_{E}(e, W)$ and $\Phi_{V}(v, W)$ be the sets of all colored flow paths in $W$ that contain the link $e$ or the node $v$, respectively. For example, $\Phi_{E}(e=(1,2), W)=\{[(1,2),(2,3)],[(1,2),(2,5)]\}$.

The spectrum of an edge $e \in E$, with respect to $\mathrm{RNF} \alpha$, is defined as:

$$
\Psi_{E}(\alpha, e) \equiv \bigcup_{w \in \Phi_{E}(e, W)} f(w)
$$

Likewise, the spectrum of a node $v$ is defined as:

$$
\Psi_{V}(\alpha, v) \equiv \bigcup_{w \in \Phi_{V}(v, W)} f(w)
$$

In Fig. 1 for instance $\Psi_{E}((1,2))=\Psi_{E}((2,4))=\Psi_{E}((2,5))=\{1\}$ and, $\Psi_{E}((1,3))=$ $\Psi_{E}((3,4))=\Psi_{E}((3,5))=\{2\}$. The spectrum of the nodes 4,5 consists of both descriptions (i.e., $\{1,2\}$ ), while the spectrum of the nodes 2,3 is $\{1\},\{2\}$ respectively.

A rainbow network flow $\alpha(W, f)$ is said to be admissible with capacity function $R$, if and only if:

$$
\left|\Psi_{E}(\alpha, e)\right|<R(e) \quad \forall e \in E
$$

The significance of this inequality is that it allows the duplication of a description by relay nodes. Therefore, two flow paths of the same color can pass through a link $e$, and yet consume 
a bandwidth of only $r$. The rainbow flow plotted in Fig. 1 is admissible because at most one description with rate $C$ is communicated over each link and the capacity of each link is $C$. This is made possible by duplicating at nodes 2,3 .

For a given $\alpha(W, f),\left|\Psi_{V}(\alpha, v)\right|$ is the number of distinct descriptions (out of a total of $K=|\chi|$ such descriptions) available to node $v$. If the node $v$ is a sink node, the reconstruction distortion at $v$ will therefore be:

$$
d_{t}=\delta\left(\left|\Psi_{V}(\alpha, t)\right|\right)
$$

The weighted average distortion at all the sink nodes is then:

$$
\bar{d}(\alpha)=|T|^{-1} \sum_{t \in T} p_{t} \delta\left(\left|\Psi_{V}(\alpha, t)\right|\right)
$$

$\mathrm{RNF}$ problem is therefore, that of finding an admissible rainbow network flow $\alpha^{*}$ that minimizes (6).

Unfortunately, RNF problem was proved to be NP-hard in its full generality $[5,6]$. For general directed acyclic graphs (DAG), the problem has an amenable integer-convex programming formulation, with a solution that can be found in polynomial time, as discussed later in this paper.

\section{Code Design}

An optimal solution to the JSNC problem requires joint optimization of the MDC code and rainbow network flow. This, however, is an extremely hard task, given that most versions of the RNF problem are intractable. In quest for a practical solution, we will resort to iterative and approximate numerical methods. We use a certain family of balanced multiple description codes that are completely parameterizable. This allows us to formulate the code design for a fixed rainbow network flow as a convex optimization problem.

\subsection{MDC using PET}

The PET technique can produce any number of balanced multiple descriptions out of a progressively encoded source stream. The idea is the following. To make $K$ balanced descriptions each of rate $r$ bits per source symbol, for a large enough value of $n$, encode $n$ samples of $X$ into a progressive bitstream $\left(b_{0}, b_{1}, \ldots, b_{n r L}\right)$, where we assume $n \cdot r$ is an integer for simplicity. Take a $K \times(n \cdot r)$ binary matrix and call it $Y=\left[Y_{i j}, i=1,2, \ldots, K, j=1,2, \ldots, n \cdot r\right]$. Now take $\mathbf{y}=\left(y_{i}, i=1,2, \ldots, K\right)$, any vector of real numbers of length $K$ such that $\sum_{i=1}^{K} y_{i}=1$. For $i=1,2, \ldots, K$ do the following: let $Y_{l}, Y_{l}^{\prime}$ for $l=1,2, \ldots, K$ be sub-matrices of $Y$ consisting of:

$$
Y_{l}=\left[Y_{i j} ; i=1: l, \quad j=ð(l-1): ð(l)\right] \text { and } Y_{l}^{\prime}=\left[Y_{i j}^{\prime} ; i=l+1: K, \quad j=ð(l-1): ð(l)\right]
$$

where $\check{\partial}(l) \triangleq \sum_{k=1}^{l} n \cdot r y_{k}$. Therefore, matrix $Y_{i}$ contains $i \cdot n \cdot r \times y_{i}$ bits while $Y_{i}^{\prime}$ has $(K-i) \cdot n \cdot r \times y_{i}$ bits. For $i=1,2, \ldots, K$, put the $i \cdot n \cdot r \cdot y_{i}$ bits of the progressive source code stream, from $b_{g(i)}$ to $b_{g(i)+i \cdot n \cdot r \cdot y_{i}}$ in $Y_{i}$, where $g(i)=\sum_{k=1}^{i} k \cdot n \cdot r y_{k}$. In $Y_{i}^{\prime}$ on the other hand, put parity symbols of a $\left(i \cdot n \cdot r \cdot y_{i}, K \cdot n \cdot r \cdot y_{i}\right)$ ideal erasure correction code corresponding to the bits in $Y_{i}$.

Now the descriptions consist of the $K$ columns of the matrix $Y$, each of $n \cdot r$ bits. The total source bits used is $n \cdot r \sum_{k=1}^{K} k y_{k}$. It is easily verified that given any $l \leq K$ descriptions, the first $\xi_{l}=\sum_{k=1}^{l} k \cdot n \cdot r \cdot y_{k}$ bits of the source bitstream can be recovered. For large enough $n$ and assuming the source is progressively refinable, given any $k$ distinct descriptions, the source can therefore be reconstructed within distortion: $D_{X}\left(\xi_{k} / n\right)=D_{X}\left(r \sum_{l=1}^{k} l y_{l}\right)$.

This is schematically depicted in bottom of Fig. 2 for a 3 -description code $(\mathrm{K}=3)$. "Grey" bits indicate source bits while "white" bits are parity symbols. The description "packets" are 
the columns of this matrix. Vectors $\mathbf{y}$ therefore parameterize the space of all MDCs that can be generated through PET. It is over this space that we will carry out our code optimization.

\subsection{Optimizing Code for a Fixed Rainbow Flow}

For any admissible flow $\alpha$, define the rainbow flow vector $(\mathrm{RFV}), \mathbf{q}(\alpha)=\left(q_{t} ; t \in T\right)$ such that:

$$
q_{t}=\left|\Psi_{V}(\alpha, v)\right|
$$

In other words, $q_{t}$ is the number of distinct descriptions available to a sink node $t$. Suppose a solution $\alpha^{*}$ is found to the RNF problem with respect to an MDC, and the rainbow flow $\alpha^{*}$ produces an RFV $\mathbf{q}^{*}$. Lets replace this MDC for which the original RNF was optimized with the MDC designed through a PET technique. Let the latter MDC be specified by the vector $\mathbf{y}$. Then, the weighted average distortion in (6), now a function of $\mathbf{q}^{*}$ and $\mathbf{y}$, can be written as:

$$
\bar{d}\left(\mathbf{y}, \mathbf{q}^{*}\right)=|T|^{-1} \sum_{t \in T} p_{t} D_{X}\left(r \sum_{l=1}^{q_{t}^{*}} l y_{l}\right)
$$

An optimal MDC can be found as an answer to the following problem:

$$
\min _{\mathbf{y} \succ 0,|\mathbf{y}|_{1}=1} \sum_{t \in T} p_{t} D_{X}\left(r \sum_{l=1}^{q_{t}^{*}} l y_{l}\right)
$$

Since $D_{X}(\cdot)$ is a convex function, this is a convex optimization problem with linear constraints and can be solved efficiently using standard tools.

\subsection{An Optimized Solution to JSNC Problem}

We can now devise a systematic procedure for finding an optimized solution to the Joint Source Network Coding problem. We approach this by first solving the RNF problem. We are particularly interested in finding the solution $\alpha^{*}$ to CRNF, because the optimization of the MDC flow is carried out regardless of the particular choice of the MDC and the statistics of the underlying source. As stated in Section 3, the goal of CRNF is to find a flow that maximizes the sum of distinct descriptions received by all the sink nodes. This approach is most reasonable when the reconstruction at all the sink nodes is equally important (e.g., $p_{t}=1, \forall t \in T$ ). Given an optimized RNF and the resulting RFV, the MDC is optimized by solving (8).

\section{Numerical Simulations}

The proposed JSNC approach is tested on a number of randomly generated directed acyclic network graphs. The simulation results are examined in this section.

\subsection{0-1 Linear Integer Programming for CRNF}

For directed acyclic graphs (DAG), the CRNF can be posed as a 0-1 linear integer programming problem. Let $\chi=\{1,2, \ldots, K\}$ be the set of all descriptions. For each edge $e=\left(v, v^{\prime}\right) \in E$, define a binary variable $x_{v, v^{\prime}}^{k}$ that is 1 if $k \in \Psi_{E}(\alpha, e)$ and 0 otherwise. Furthermore, for each node $v$, we use a binary variable $y_{v}^{k}$ to indicate whether $k \in \Psi_{V}(\alpha, v)$, that is, whether description $k$ is received by $v$. For $v \in S$ that is a source of description $k, y_{v}^{k}=1$ automatically. For $v$ that is not a source of the color $k$, obviously

$$
y_{v}^{k} \leq \sum_{v^{\prime} \in ß(v)} x_{v, v^{\prime}}^{k}
$$




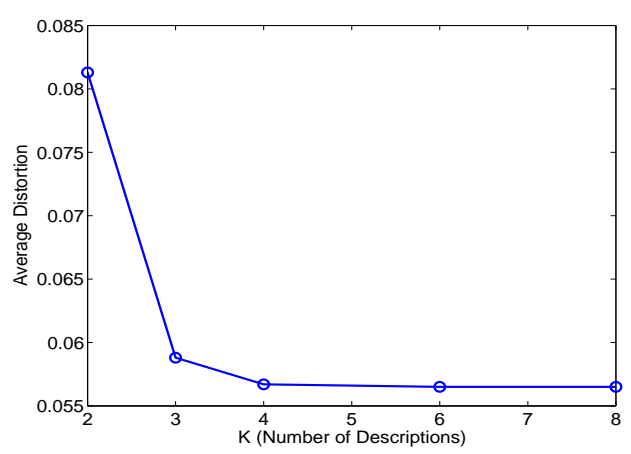

Figure 3: Distortion as a function of the number of descriptions. Description rate is $r=1$, the network has $N=50$ nodes and $C_{\max }=3$ and $m=3$.

where $B(v)$ is the set of all nodes that have a link into $v$.

A relay node can duplicate descriptions it receives from other nodes, hence

$$
\forall j \quad x_{i, j}^{k} \leq y_{i}^{k} .
$$

The edge capacity constraint is:

$$
\forall e=\left(v, v^{\prime}\right) \in E, \quad r \sum_{k} x_{v, v^{\prime}}^{k} \leq R(e)
$$

CRNF problem for DAG reduces to finding the binary variables $x_{v, v^{\prime}}^{k}$ that that maximizes the sum of the number of distinct descriptions received by all the sink nodes, that is, $\sum_{t \in T} y_{t}^{k}$. For a fixed number of descriptions $K$ and rate $r$, this is a linear binary program with linear constraints which is solved numerically.

\subsection{Network Simulation Setup}

We produce a family of DAGs motivated by a simplistic model for the growth of peer-to-peer networks. We start from a single node. At each step, a new node is added. Then $m$ nodes are chosen at random with replacement from the existing nodes, and a link is made from each of the $m$ chosen nodes to the new node. Once the network grows to $N$ nodes, we assign a capacity $C(e)$ to each link $e$, where $C(e)$ is a random integer between 1 and $C_{\max }$ for some choice of maximum capacity $C_{\max }$. For integer edge capacities, the optimum solution to the CRNF problem will consist of all integer flows. We use an iid Gaussian source of variance one in all cases.

We take the rate of the descriptions to be 1 . It can also be shown that for a fixed source and network, increasing the number of descriptions $K$ will not increase the overall distortion but the running time of the integer programming will increase exponentially in $K$. In this consideration, we always start with the smallest possible $K$, and increase $K$ until the overall distortion does not decrease any more.

For a network of size $N=50$, with nodes of in-degree $m=3$ and $C_{\max }=3$, the overall distortion $\bar{d}$ is optimized by first solving the instance of CRNF problem and then optimizing the MDC. The optimization is repeated for increasing number of descriptions $K$. The distortion converges to its final value for $K=6$ as shown in Fig. 3. The overall optimization took less than 5 seconds.

To see the effect of enlarging the network, we have provided simulation results for fixed $K=6, C_{\max }=3$ and $m=3$ and different networks sizes. For the above family of random networks, the increase in the network size will offer greater path diversity to the more recently 


\begin{tabular}{|c|c||c|c|c|c|c|c|}
\hline $\mathrm{N}$ & $\bar{d}^{*}$ & $y_{1}$ & $y_{2}$ & $y_{3}$ & $y_{4}$ & $y_{5}$ & $y_{6}$ \\
\hline \hline 50 & 0.1104 & 0.0 & 0.7069 & 0.2067 & 0.083 & 0.0 & 0.0 \\
\hline 100 & 0.0466 & 0.0 & 0.445 & 0.070 & 0.396 & 0.090 & 0.0 \\
\hline 200 & 0.0302 & 0.0 & 0.204 & 0.556 & 0.094 & 0.117 & 0.030 \\
\hline
\end{tabular}

Table 1: The optimal vector $\mathbf{y}$ for different network sizes. $C_{\max }=3, K=6$ and $m=3$.

created nodes which are at the bottom of the network hierarchy. Therefore, as the network size grows, the fraction of nodes that receive higher number of descriptions increases which leads to a decrease in the overall average distortion.

\section{Conclusion}

We introduced the problem of joint network-source coding (JNSC) in which the goal is to best communicate a real valued source to a number of destinations using the collaboration of all nodes in the network. We found that multiple description coding is a powerful tool for exploiting path diversity in a network. We provided a systematic approach for optimizing the routing of descriptions as well as optimally designing the MDC. To our best knowledge, this is the only known formulation and systematic approach to joint network-source coding problem.

Our ongoing research is on an iterative optimization approach in which, (1) optimize the flow of descriptions for a given MDC and, (2) optimize in turn the MDC with respect to the resulting flow, (3) continue the process iteratively until there is no further reduction in the average distortion.

We are also applying our approach to a peer-to-peer networking scenario in which the goal is to have a real-time presentation of a multimedia content in the network. In this case, maximizing the description diversity has to be done locally at individual nodes, and the network dynamics should also be taken into account.

\section{References}

[1] R. Ahlswede, N. Cai, S.-Y. R. Li and R. W. Yeung, "Network information flow," IEEE Trans. on Information Theory, vol. 46, Jul 2000.

[2] [9] L. H. Ozarow. On a Source Coding Problem with Two Channels and Three Receivers. Bell System Technical Journal, 59(10), 1980

[3] M. F. Barnsley, R. L. Devaney, B. B. Mandelbrot, H. Peitgen, D. Saupe, and R. F. Voss. The Science of Fractal Images. Springer-Verlag, 1988

[4] S. Boucheron and M. R. Salamatian, About Priority encoding transmission, IEEE Trans. on Information Theory 46, Mar. 2000.

[5] X. Wu, Bin Ma, N. Sarshar, "Rainbow Network Problems and Multiple Description Coding", IEEE International Symposium on Information Theory, ISIT05, Pages:268 272.

[6] B. Ma and X. Wu, Maximum network flow of multiple description codes, Proc. of NetCod 2005, Apr. 2005. 